Bol. Soc. Вот. México 36:71-76, 2 figs. (1972)

\title{
UNA NUEVA FRANKENIACEAE DEL NORTE DE MEXICO
}

\author{
por Francisco González Medrano*
}

Durante el curso de una excursión por el altiplano mexicano colecté dentro de una comunidad de halófitas, una planta que destacaba por su hábito juniperoide. Después de un detallado estudio llegué a la conclusión que se trataba de una especie nueva del género Frankenia L., la que describo a continuación:

Frankenia margaritae. González Medrano, sp. nov.

Suffrutex adscendens 10-15 cm. altus, in diametro usque ad $27 \mathrm{~cm}$. Ramuli subquadrangulares. Folia subprismatica, $3-4 \mathrm{~mm}$. longa, $1 \mathrm{~mm}$. lata, marginibus valde revolutis itaque pagina inferior laminae omnino occulta. Sepala 4 basin versus connata, 4.5-5.5 mm. longa. Petala 4, alba rosaceave, spathulata, $6 \cdot 7 \mathrm{~mm}$. longa, trientem superiorem versus $1.5-2 \mathrm{~mm}$. lata sed infra angustata, lamina ligulam minutam $3 \mathrm{~mm}$. infra apicem obtusum efferenti. Stamina 4, inaequalia, duo longiora, $5.5-6 \mathrm{~mm}$. longa, duo breviora, $4.5-5 \mathrm{~mm}$. longa. Ovarium glabrum elongatum 1.8-2 mm. longum, e tribus carpellis compositum sed uniloculare. Stigma indivisum, obovatum. Ovulum unicum amphitropum in funiculum $2 \mathrm{~mm}$. longo portatum.

Planta subarbustiva ascendente, de 10 a $15 \mathrm{~cm}$. de alto, con ramas de 8 a $10 \mathrm{~cm}$. de largo. Tallo híspido con pelos simples esparcidos; entrenudos de 5 n!m. de largo; hojas marcadamente revolutas, subprismáticas de 3 a $4 . \mathrm{mm}$. de largo por $1 \mathrm{~mm}$. de ancho, márgenes híspidas hacia el tercio superior, la parte inferior glabra, base cordada o subsagitada; márgenes revolutas con la lámina de la cara inferior totalmente oculta, con un surco medio muy notable, los bordes superiores cóncavos.

Flores en las axilas de las hojas, terminales o subterminales; el cáliz con pubescencia escabrosa en las márgenes, sépalos 4. unidos hacia la base, de 4.5 a $5.5 \mathrm{~mm}$. de largo $(5.2 \mathrm{~mm}$. de promedio) por $1.5 \mathrm{~mm}$. de ancho, con 4

* Jardín Botánico U.N.A.M. actualmente Departamento de Botánica, Instituto de Biología, U.N.A.M. 
lóbulos más o menos triangulares de $1 \mathrm{~mm}$. de longitud. Pétalos 4 , blancos o rosados y más largos que los sépalos, de 6 a $7 \mathrm{~mm}$. de liargo, de 1.5 a $2 \mathrm{~mm}$. de ancho en su tercio superior, angostados hacia la base y de forma espatulada, de ápice obtuso, con una lígula pequeña en cada pétalo situada a unos $3 \mathrm{~mm}$. abajo del ápice.

Estambres 4, con los filamentos aplanados, adosados al ovario y al estilo, de los 4 estambres 2 son más pequeños en longitud (4.5 a $5 \mathrm{~mm}$.) y 2 más largos (5.5 a $6 \mathrm{~mm}$.), anteras con dehiscencia longitudinal de $1 \mathrm{~mm}$. de largo por $0.8 \mathrm{~mm}$. de ancho.

Ovario glabro, alargado de 1.8 a $2 \mathrm{~mm}$. de largo, estilo indiviso de 4 a $4.5 \mathrm{~mm}$. de longitud, estigma obovado; tricarpelar, unilocular con un solo óvulo naciendo de una placenta basal, con un funículo largo $(2 \mathrm{~mm}$.) y el óvulo anfítropo.

MEXICO: Nuevo León. 20 kms. al sur de San Roberto, Municipio San José de Raíces, 20 de mayo de 1966, abundante en una depresión salina, F. González Medrano 1337 (Holotipo MEXU, Isotipos en GH, RSA, TEX y UC). 32 Kms. al sur de San Roberto, A. López. L. 199. (MEXU); 10 Kms. adelante de Puerto de Nopalillo (a los lados de la nueva carretera MatehualaSaltillo) J. Valdés, E. Hernández Xolocotzi y F. Miranda, A-78 (MEXU); 12-13 kms. al sur de San José de Raíces, F. González Medrano, M. Sousa y C. Delgadillo, 1751. (MEXU).

Esta especie perteneciente a la sección Basigonia, Niedenz. del género, es muy afín a Frankenia palmeri S. Watson. y a $F$. gypsophila Johnston. de Norteamérica, de las que difiere por su mayor porte, además del carácter indiviso del estilo y la posesión de un óvulo. El hábito juniperoide de $F$. gypsophila y F. margaritae sugiere también cierta relación con algunas especies australianas y sudamericanas del género.

Esta especie crece en la localidad tipo asociada con $F$. gypsophila Johnston.

Clave para la separación de las tres especies consideradas:

Estilo indiviso; con un solo óvulo en toda la cavidad del ovario, sostenido por un funículo largo $(2 \mathrm{~mm}$. $) \ldots \ldots \ldots \ldots \ldots$. margaritae.

Estilo dividido en 2 o 3 ramas, óvulo 2 o más en toda la cavidad del ovario sostenidos por funículos cortos (menos de 2 mm.)

Cáliz de $3 \mathrm{~mm}$. de largo, pétalos lineares del 1.5 a $2 \mathrm{~mm}$. de largo, estilo con 2 ramas ....................... palmeri. 
Cáliz de 3.4 a $5 \mathrm{~mm}$. de largo, pétalos angostamente oblanceolados de $5 \mathrm{~mm}$. de largo, estilo con 3 ramas (excepcional-

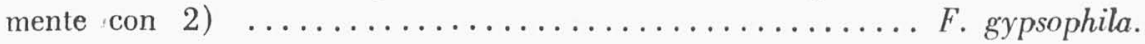

El nombre de esta especie está dedicada a la señora Biól. Margarita Hernández de González, esposa del autor, por toda la ayuda moral y material brindada durante el desarrollo de mi trabajo.

Agradezco a las doctoras Elizabeth A. Shaw y Bernice G. Schubert la traducción de la descripción al latín; al M. en C. Claudio Delgadillo su colaboración en la consecución del material bibliográfico y a los doctores A. Gómez-Pompa y Lorin Nevling Jr., sus valiosas sugestiones.

P.D.

Cuando el autor había entregado este trabajo para su publicacion al Comité Editorial de la Sociedad Botánica de México, recibió la noticia que el I)r. B. L. Turner, de la Universidad de Texas en Austin, estaba trabajando en Frankenia. El Dr. Turner conociendo que en México se estaban estudiando Jas especies de este género, escribió al Dr. A. Gómez-Pompa para informarse sobre el estado de las investigaciones, ya que él había encontrado una especie uneva muy interesante para el entendimiento de la evolución del género. Hahiéndole enviado al Dr. Turner copia de nuestro manuscrito, coincidió que Frankenia margaritae era la misma especie que él había estado estudiando y que pensaba publicar. Habiéndose enterado el Dr. Turner de esta coincidencia, amablemente nos proporcionó el esquema que acompaña a esta descripción, dándonos además una lección de ética profesional. Que sean estas lineas una muestra de agradecimiento.

\section{RESUMEN}

Se describe una nueva especie de Frankeniaceae: Frankenia margaritae, con material proveniente del norte de México. Esta especie se encuentra relacionada con Frankenia gypsophila Johnston, del altiplano mexicano y con Frankenia palmeri S. Watson, de Baja California y Sonora (Méx.) y California (EE. UU.).

Se presenta una clave artificial para separar las tres especies involucradas. 
BOLETIN DE LA SOCIEDAD BOTANICA DE MEXICO No. 32, 1972

\section{SUMMARY}

A new species of Frankeniaceae, Frankenia margaritae is described from northern Mexico. Its relationship with $F$. gypsophila Johnston and $F$. palmeri $\mathrm{S}$. Watson, is discussed; a systematic key to the closely related species is given. 


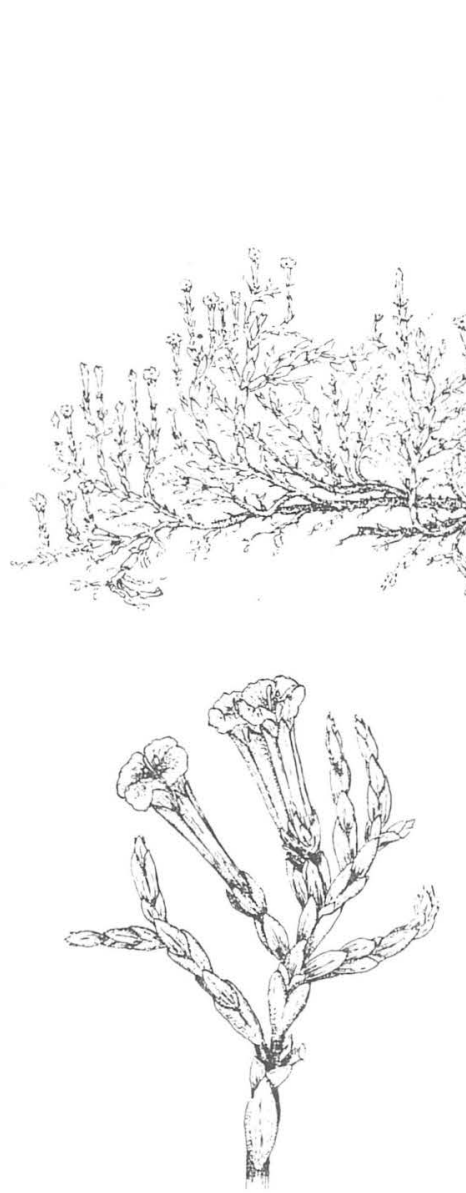

A
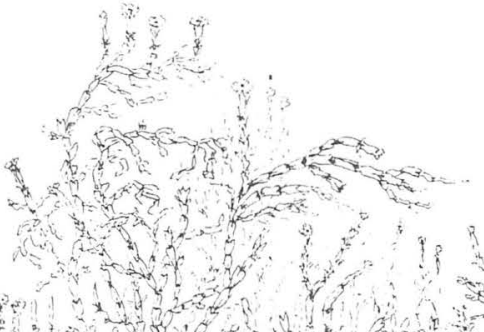

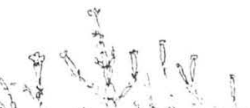

${ }^{3}=5+2$

I) $x+3203$
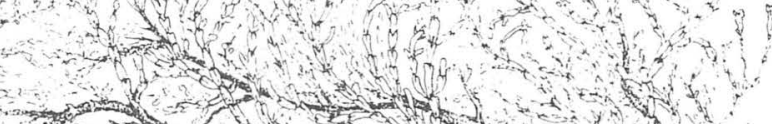

$x$.

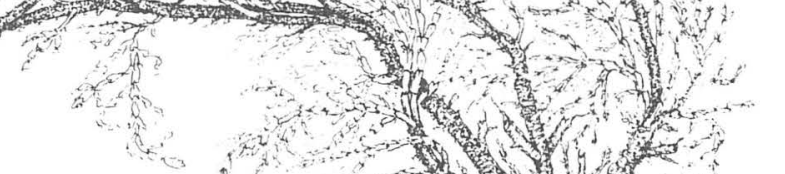

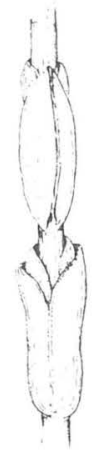

B

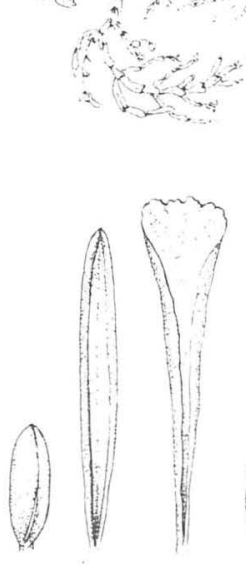

C

D

E

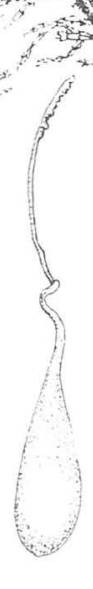

F

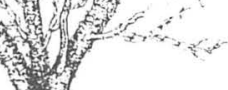

(a)

a

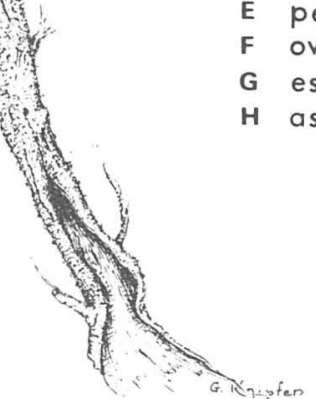

H
A rama florifera
B implantación hojas
C hoja: cara abaxial
D sépalo
E pétalo: vista ventral
G estambre
H aspecto vegetativo

Bacc.

$1115(T=x)$ 


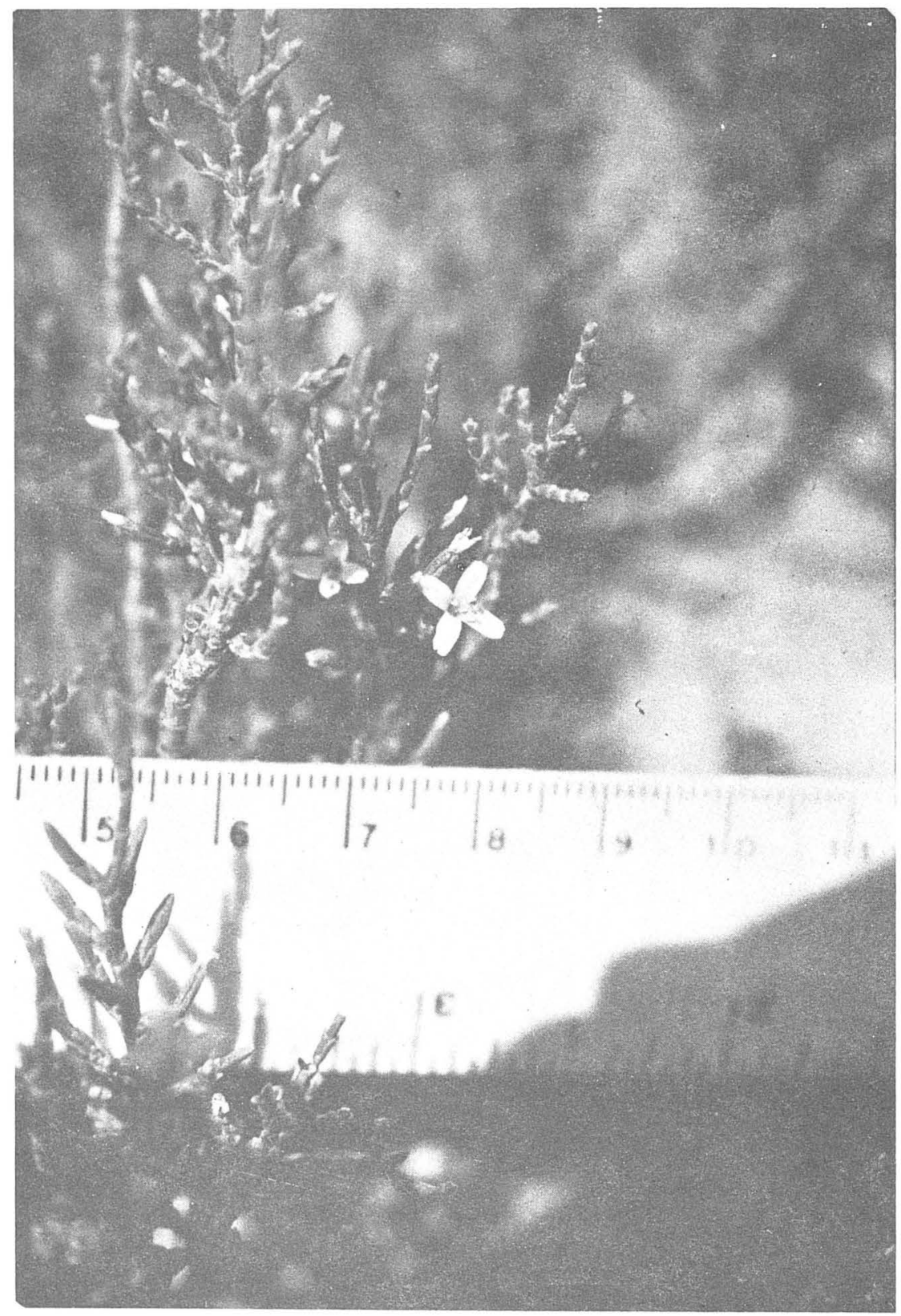

